ORIGINAL ARTICLE

\title{
Isolation of cytoplasmic NADPH-dependent phenol hydroxylase and catechol-1,2- dioxygenase from Candida tropicalis yeast
}

\author{
Lenka VILÍMKOVÁ ${ }^{1}$, Jan PÁCA Jr ${ }^{1}$, Veronika KREMLÁČKOVÁ ${ }^{1}$, Jan PÁCA ${ }^{2}$, Marie STIBOROVÁ ${ }^{1}$ \\ 1 Department of Biochemistry, Faculty of Science, Charles University, Prague, Czech Republic \\ 2 Department of Fermentation Chemistry and Bioengineering, Institute of Chemical Technology, Prague, Czech Republic
}

\section{ABSTRACT}

ITX013408A04 • Received:21 November 2008 • Revised:25 November 2008 • Accepted: 7 December 2008 • Electronic Publication: November 2010

The efficiencies of NADPH-dependent phenol hydroxylase (EC 1.14.13.7) and catechol 1,2-dioxygenase (EC.1.13.11.1) in biodegradation of phenol in the cytosolic fraction isolated from yeast Candida tropicalis were investigated. Enzymatic activities of both NADPHdependent phenol hydroxylase and catechol 1,2-dioxygenase were detected in the cytosolic fraction of C. tropicalis grown on medium containing phenol. Using the procedure consisting of chromatography on DEAE-Sepharose, fractionation by polyethylene glycol 6000 and gel permeation chromatography on Sepharose 4B the enzyme responsible for phenol hydroxylation in cytosol, NADPHdependent phenol hydroxylase, was isolated from the cytosolic fraction of C. tropicalis close to homogeneity. However, fractionation with polyethylene glycol 6000 lead to a decrease in catechol 1,2-dioxygenase activity. Therefore, another procedure was tested to purify this enzyme. Gel permeation chromatography of proteins of the eluate obtained by chromatography on a DEAE-Sepharose column was utilized to separate phenol hydroxylase and catechol 1,2-dioxygenase. Among gel permeation chromatography on columns of Sephadex G-100, Sephacryl S-300 and Sepharose 4B tested for their efficiencies to isolate phenol hydroxylase and catechol 1,2-dioxygenase, that on Sephacryl S-300 was found to be suitable for such a procedure. Nevertheless, even this chromatographic method did not lead to obtain catechol 1,2-dioxygenase in sufficient amounts and purity for its further characterization. The data demonstrate the progress in resolving the enzymes responsible for the first two steps of phenol degradation by the C. tropicalis strain.

KEY WORDS: environmental pollutants; phenol; biodegradation; yeast; Candida tropicalis; NADPH-dependent phenol hydroxylase; catechol-1,2-dioxygenase

\section{Introduction}

Phenol and its derivatives are found in wide variety of wastewaters including those from the oil refining, petrochemical, coke and coal gasification industries. Removal of phenol from such wastewaters can be achieved through aerobic biodegradation in well-run activated sludge plants. Pseudomonas is a bacterial genus commonly found in such plants and Pseudomonas putida is a species capable of using phenol as a major source (Bayly and Wigmore, 1973; Yang and Humphrey, 1975). In addition, several other mesophilic bacteria are able to degrade phenol, including Alcaligenes spp. and Spreptomyces setonii and also the thermophile, Bacillus stearothermophilus (Gurujeyalakshmi and Oriel,

Correspondence address:

\section{Prof. Marie Stiborová, DrSc.}

Department of Biochemistry, Faculty of Science, Charles University, Prague, Albertov 2030, 12840 Prague 2, Czech Republic

E-MAlL: stiborov@natur.cuni.cz
1989). Although bacteria are most likely to be responsible for aerobic breakdown of phenol in activated sludge, fungi including Trichosporon cutaneum, Candida albicans TL3 and Candida tropicalis are also capable of utilizing phenol as the major carbon source (Krug et al., 1985; Krug and Straube, 1986; Chang et al., 1998; Bastos et al., 2000; Komárková and Páca, 2000; Páca et al., 2002; Komárková et al., 2003; Stiborová et al., 2003; Ahuatzi-Chacon et al., 2004; Tsai et al., 2005). The aerobic degradation pathways in bacteria and yeast involve the occurrence of vicinal diols as substrates of ring-cleaving enzymes. Thus, the first step of phenol degradation is a hydroxylation of phenol to catechol (Figure 1). Catechol can undergo fission either by an intradiol or an extra-diol type of cleavage (ortho- or meta-fission). Meta-fission leads to 2-hydroxymuconic semialdehyde and further to formate, acetaldehyde, and pyruvate. Such a catechol cleavage was not found in yeast. Ortho-fission, found in yeast such as T. cutaneum, C. albicans TL3 and C. tropicalis, gives rise to cis, cis-muconic acid (Figure 2), which is converted in further enzymatic steps via 3-oxoadipate to succinate and acetyl-CoA. These products enter the central metabolism of the cell (Krug et al., 1985; Krug and Straube, 
1986; Bastos et al., 2000; Komárková and Páca, 2000; Páca et al., 2002; Komárková et al., 2003; Ahuatzi-Chacon et al., 2004; Tsai et al., 2005). Although examples are known in which the yeast $C$. tropicalis utilizes phenol for growth or metabolism (Krug et al., 1985; Krug and Straube, 1986; Stephenson, 1990; Chang et al., 1998; Bastos et al., 2000; Komárková and Páca, 2000; Páca et al., 2002; Komárková et al., 2003; Ahuatzi-Chacon et al., 2004) much less information on the nature of the phenol-oxidizing enzymes in this microorganism are known.

The enzymes responsible for the first step of degradation (the formation of catechol: Figure 1) in C. tropicalis yeast are: (i) cytochrome P450 (EC 1.14.15.1), the enzyme of the mixed function monooxygenase system localized in the membrane of endoplasmic reticulum (Stiborová et al., 2003; 2004) and (ii) cytoplasmic NADPH-dependent phenol hydroxylase (EC 1.14.13.7) (Krug et al., 1085; Krug and Straube, 1986; Xu et al., 2000; Stiborová et al., 2004; Páca et al., 2007). Indeed, recently, we have found that microsomal cytochrome P450 and cytosolic NADPH-dependent phenol hydroxylase are expressed in C. tropicalis grown on phenol and are capable of hydroxylation of phenol to form catechol (Stiborová et al., 2003; Páca et al., 2007). Cytosolic NADPH-dependent phenol hydroxylase seems to be the predominant enzyme responsible for the first step of phenol biodegradation in the $C$. tropicalis yeast; its activity is more than two orders of magnitude higher than that found in the microsomal fraction of this microorganism (Stiborová et al., 2003; Páca et al., 2007). During the second step of phenol degradation in Candida yeast, intra-diol cleavage of catechol to cis,cis- muconic acid (Figure 2) occurs (Bastos et al., 2000; Páca et al., 2002; A huatzi-Chacon et al., 2004; Tsai et al., 2005; 2007), being catalyzed by cytosolic catechol 1,2-dioxygenase (EC.1.13.11.1), the enzyme found in several microorganisms, including yeast (Nakai et al., 1990; Eck and Bettler, 1991; Briganti et al., 1997; Shen et al., 2004; Tsai and Li, 2007). NADPH-dependent phenol hydroxylase has already been purified from the cytosolic fraction of $C$. tropicalis.and partially characterized (Páca et al., 2007). However, information on catechol 1,2-dioxygenase of this microorganism are scarce. Even though the activity of this enzyme was detected in C. tropicalis cytosol (AhuatziChacon et al., 2004), its isolation from this microorganism has not been described as yet.

The aim of the present study was to develop the procedure to isolate both phenol hydroxylase and catechol 1,2-dioxygenase from the cytosolic fraction of C. tropicalis.

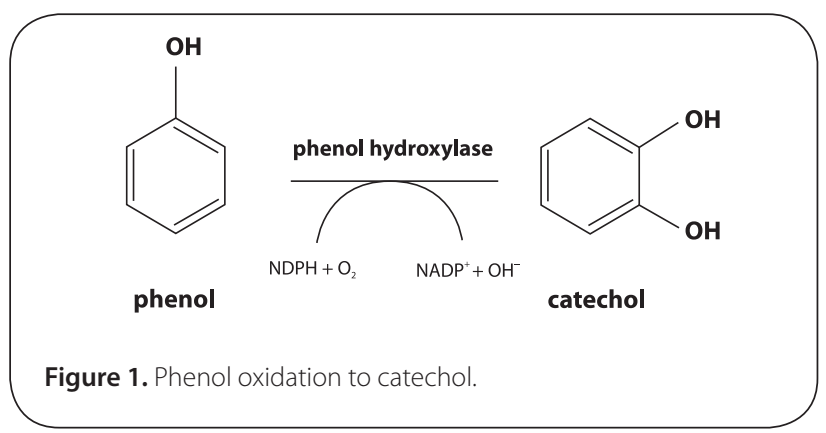

\section{Materials and methods}

\section{Chemicals}

Chemicals were obtained from the following sources: $\mathrm{NADPH}$, catechol and bicinchoninic acid (2,2'-biquinoline4,4'-dicarboxylic acid) from Sigma Chemical Co., (St. Louis, MO), DEAE-Sepharose, Sephacryl S-300, Sephadex G-100 and Sepharose 4B from Pharmacia (Uppsala). Other chemicals were supplied by Lachema (Brno). All chemicals were of reagent grade purity or better.

\section{Microorganisms, cultivation methods and preparation of microsomal and cytosolic fractions}

The yeast C. tropicalis was isolated from soil contaminated with aromatic hydrocarbons and identified using the culture collection and Research Center (Brno, Czech Republic) (Komárková and Páca, 2000). The yeast culture was maintained on slope agar with mineral salts and glucose as a carbon and energy source at $4^{\circ} \mathrm{C}$. The growth medium was BSM medium $\left[4.3 \mathrm{~g} / 1 \mathrm{~K}_{2} \mathrm{HPO}_{4}, 3.4 \mathrm{~g} / 1 \mathrm{KH}_{2} \mathrm{PO}_{4}, 2 \mathrm{~g} / 1\right.$ $\left.\left(\mathrm{NH}_{4}\right)_{2} \mathrm{SO}_{4}, 0.34 \mathrm{~g} / \mathrm{l} \mathrm{MgCl} 2.6 \mathrm{H}_{2} \mathrm{O}\right]$ containing $350 \mathrm{~g} / \mathrm{l}$ phenol as a sole carbon and energy source (phenol medium). Cell cultivations were carried out in shaking flasks using fed batch process with the growth medium containing phenol (see above) at $30^{\circ} \mathrm{C}$ and pH5.2 as described previously (Martius et al., 1996; Páca and Martius, 1996; Stiborová et al., 2003; Páca et al., 2007).

After separation, the cells were washed three times with distilled water and disintegrated using mechanical disruption of the cells in the presence of liquid nitrogen to obtain the cell-free homogenate. The isolation of the microsomal and cytosolic fractions from the $C$. tropicalis cell-free homogenate was carried out by differential centrifugation (Stiborová et al., 2003; Páca et al., 2007) by the procedure used for isolation of such subcellular fractions from rat liver (Stiborová et al., 1995, 2001a;b).

\section{Purification of NADPH-dependent phenol hydroxylase and catechol 1,2-dioxygenase from C. tropicalis cytosol}

All operations were carried out at $4{ }^{\circ} \mathrm{C}$. For the first, the procedure which was used in our former study to isolate NADPH-dependent phenol hydroxylase was used (Páca et al., 2007). Briefly, cytosolic fraction of C. tropicalis $(330 \mathrm{ml})$ was applied to a DEAE-Sepharose column $(2.6 \times 22 \mathrm{~cm})$ equilibrated with $50 \mathrm{mM}$ sodium phosphate buffer $\mathrm{pH}$ 7.6. The NADPH-dependent phenol hydroxylase was eluted using a linear gradient of $0-0.5 \mathrm{M} \mathrm{NaCl}$ in the same

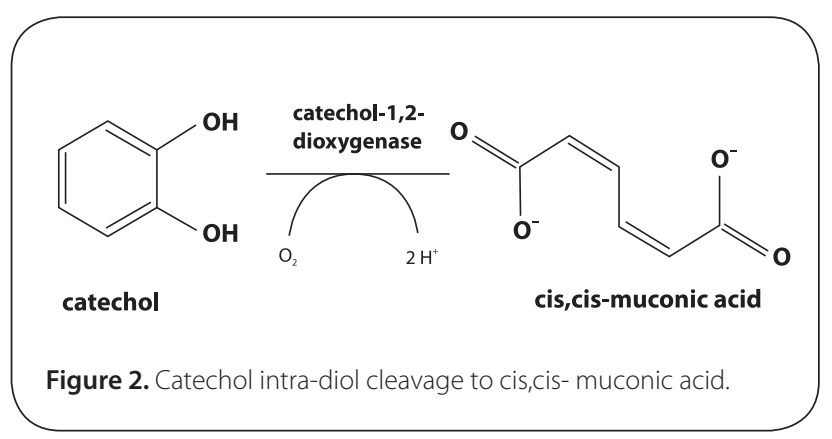


buffer. Phenol hydroxylase eluted at $0.1-0.15 \mathrm{M}$. Fractions containing phenol hydroxylase activities were pooled and precipitated by PEG 6000 (16\% saturation). The precipitate was dissolved in $50 \mathrm{mM}$ sodium phosphate buffer $\mathrm{pH} 7.6$ $(2 \mathrm{ml})$ and applied to a Sepharose 4B column $(1.6 \times 60 \mathrm{~cm})$, previously equilibrated with the same buffer. The enzyme was eluted using $50 \mathrm{mM}$ sodium phosphate buffer pH 7.6 and the fractions containing the phenol hydroxylase activity were pooled, frozen and stored at $-20^{\circ} \mathrm{C}$ until used.

However, using precipitation of proteins with PEG 6000 (16\% saturation), no catechol 1,2-dioxygenase activity was detectable. Therefore, proteins of eluate obtained by DEAESepharose chromatography were lyophilized and enzymes additionally purified by gel permeation chromatography on columns $(0.5 \times 50 \mathrm{~cm}$, bed volume $20 \mathrm{ml})$ of Sephadex G-100, Sephacryl S-300 and Sepharose 4B, equilibrated with $50 \mathrm{mM}$ sodium phosphate buffer $\mathrm{pH}$ 7.6. Lyophilized proteins $(2 \mathrm{mg})$, re-suspended in $200 \mu \mathrm{l}$ of $50 \mathrm{mM}$ sodium phosphate buffer (pH7.6), were applied on a column and eluted with the same buffer.

The phenol hydroxylase and catechol 1,2-dioxygenase activities were followed by formation and consumption of catechol, respectively, measured with HPLC, using a column of Nucleosil 100-5 C18 $(4 \times 250 \mathrm{~mm})$ as described previously (Stiborová et al., 2003; Páca et al., 2007). The major product formed by phenol hydroxylation was identified by comparison of its retention time with an authentic standard of catechol, having the retention time of $7.9 \mathrm{~min}$ and by mass and UV/vis absorbance spectroscopy. Mass spectra were recorded on a FINNIGAN MAT INCOS 50 (electron impact, $70 \mathrm{eV}$, low resolution, direct inlet). UV/vis spectra were recorded on a Hewlett-Packard 8453 diode array spectrophotometer (Stiborová et al., 2003; Páca et al., 2007).

\section{Results}

The cytosolic fraction of C. tropicalis is able to oxidize phenol; a time-dependent decrease in phenol followed by an increase in formation of an oxidation metabolite, catechol, was found (data not shown). Non-Michaelian saturation curves were seen when the initial velocity of phenol oxidation catalyzed by the crude cytosolic fraction was plotted as a function of phenol concentrations (Figure 3A,B). The observed decrease in amounts of catechol generated in incubations containing the crude cytosolic fraction (Figure 3B), might be caused by its consumption with catechol 1,2-dioxygenase, whose activity was found by Ahuatzi-Chacon and collaborators (2004) in C. tropicalis cytosol. Therefore, these findings suggest the presence not only of NADPH-dependent phenol hydroxylase, but also catechol 1,2-dioxygenase in this subcellular fraction.

Using the procedure described in our previous work (Páca et al., 2007), consisting of the chromatography on a DEAE-Sepharose column, fractionation with PEG 6000 and a gel filtration on Sepharose 4B (Table 1A), NADPHdependent phenol hydroxylase was purified from the cytosolic fraction of $C$. tropicalis close to homogeneity. Figure 4 shows the kinetics of phenol oxidation by the

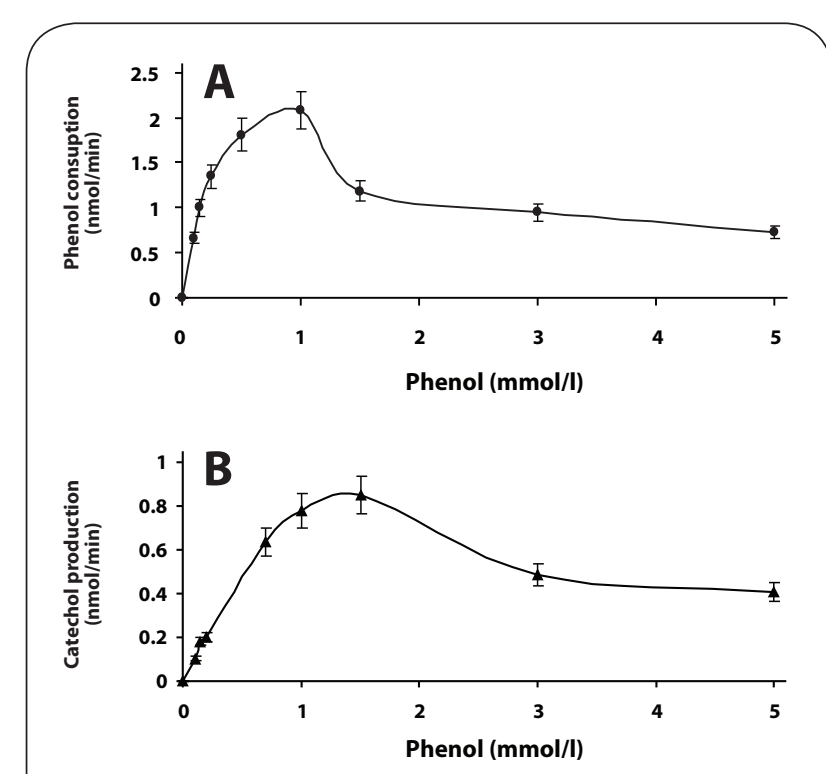

Figure 3. Substrate affinities of cytosolic enzymes of $\mathbf{C}$. tropicalis towards phenol measured by disappearance of phenol (A) and formation of catechol (B)

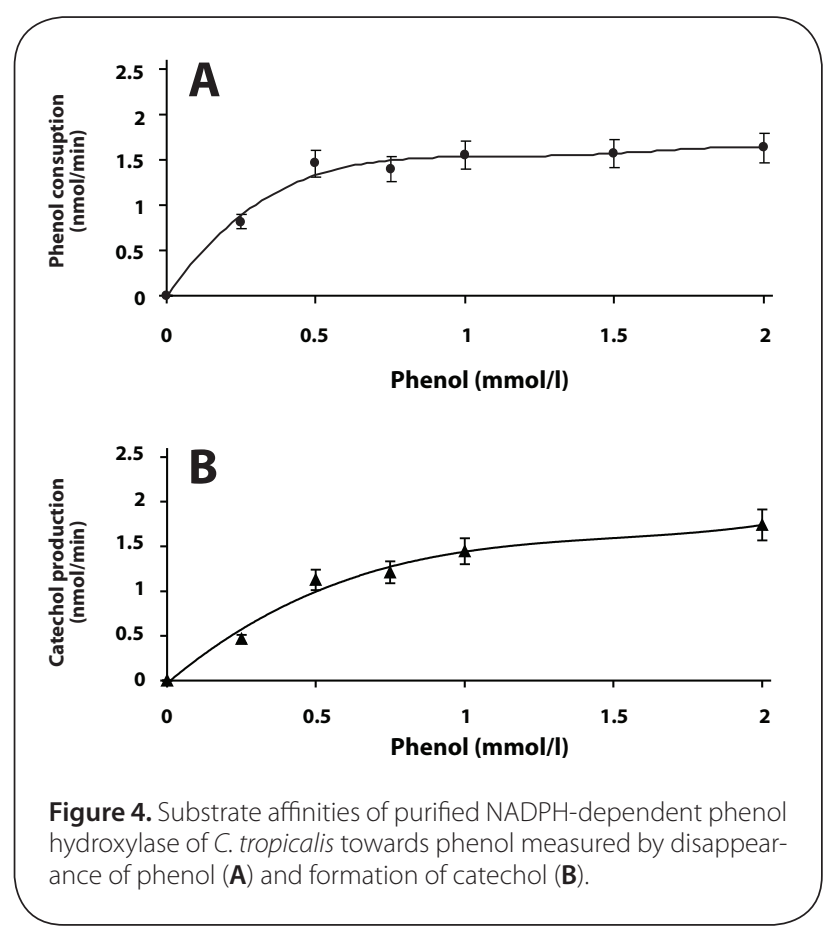

purified enzyme. Phenol consumption and formation of catechol was measured in the reaction medium, which contained isolated NADPH-dependent phenol hydroxylase, $\mathrm{NADPH}$ and various concentrations of phenol. The reaction measured by both phenol consumption and formation of catechol followed the Michaelis-Menten kinetics (Figure 4). The Michaelian kinetics measured by formation of catechol confirms that the preparation of purified phenol hydroxylase is free of catechol 1,2-dioxygenase utilizing catechol as substrate. The values of a maximal velocity $\left(\mathrm{V}_{\max }\right)$ and an 
Table 1.

Purification of NADPH-dependent phenol hydroxylase and catechol 1,2-dioxygenase from C. tropicalis.

A

\begin{tabular}{lcccc} 
A Fraction & Volume & Proteins & & Specific activity \\
& & & phenol hydroxylase & catechol 1,2-dioxygenase \\
(nmol catechol/min/mg) & not measured \\
Cytosol & $(\mathrm{ml})$ & $(\mathrm{mg} / \mathrm{ml})$ & 2.1 & 23.1 \\
Eluate, DEAE-Sepharose & 330.0 & 2.16 & 28.4 & 32.5 \\
PEG 6000, ppt ${ }^{\mathrm{a}}$ 0-16\% & 70.0 & 1.73 & 41.5 & not detectable \\
Eluate, Sepharose 4B & 5.2 & 4.14 & 1.15 & not detectable \\
\hline
\end{tabular}

appt - precipitate

B

phenol hydroxylase

(nmol phenol/min/mg) catechol 1,2-dioxygenase

(nmol catechol/min/mg)

not measured

23.1

not measured

not measured

14.8

54.8

45.9

100.0

30.0

110.8

Experimental conditions are described in the Material and methods section.

apparent Michaelis constant $\left(\mathrm{K}_{\mathrm{m}}\right)$ for oxidation of phenol are $54.3 \mathrm{nmol} / \mathrm{min}$ per $\mathrm{mg}$ of protein and $0.45 \mathrm{mmol} / \mathrm{l}$, respectively. The values of $\mathrm{V}_{\max }$ and an apparent $\mathrm{K}_{\mathrm{m}}$ for oxidation of phenol determined from the formation of catechol are $54.4 \mathrm{nmol} / \mathrm{min}$ per $\mathrm{mg}$ of protein and $0.45 \mathrm{mmol} / \mathrm{l}$, respectively.

While NADPH-dependent phenol hydroxylase and catechol 1,2-dioxygenase activities were detectable in the eluate obtained by the chromatography on a DEAESepharose column, no activity of catechol 1,2-dioxygenase was detectable if proteins were precipitated with PEG 6000 (Table 1A). Therefore, another procedure was tested to obtain the enzymatically active catechol 1,2-dioxygenase. The proteins of pooled fractions containing phenol hydroxylase and catechol 1,2-dioxygenase activities, obtained by chromatography on DEAE-Sepharose, were lyophilized and used for additional enzyme purification. Gel permeation chromatography on columns of Sephadex G-100, Sephacryl S-300 and Sepharose 4B was utilized to separate phenol hydroxylase and catechol 1,2-dioxygenase (Figure 5). Using these procedures, besides protein fractions containing phenol hydroxylase and catechol 1,2-dioxygenase, the fraction of proteins having a lower molecular mass than both enzymes was eluted as a distinguish peak (Figure 5). Such a separation of ballast proteins led to an increase in specific activities of both enzymes (Table 1B). However, different efficiencies to separate phenol hydroxylase and catechol 1,2-dioxygenase were found for individual chromatography.
The separation of both enzymes was reached only using chromatography on a column of Sephacryl S-300, whereas chromatography on Sephadex G-100 and Sepharose 4B was ineffective under the conditions used in the experiments.

\section{Discussion}

Several fungi including $C$. tropicalis are capable of utilizing phenol as the sole carbon and energy source. These organisms might therefore be useful for biotechnological applications such as decontamination of phenol in wastewaters. The hydroxylation of phenol to catechol, and its additional intra-diol cleavage are the initial and rate-determining steps in the phenol degradation pathways in Candida yeast. The question which of the yeast enzymes are responsible for these two steps of phenol degradation in C. tropicalis yeast has not been fully answered yet.

Here, we demonstrate that NADPH-dependent phenol hydroxylase capable of oxidation of phenol to catechol and catechol 1,2-dioxygenase are present in cytosol of $C$. tropicalis yeast. Non-Michaelian saturation curves were seen when the initial velocity of phenol oxidation catalyzed by the crude cytosolic fraction was plotted as a function of phenol concentrations. Here, we show that the observed decrease in amounts of catechol generated in incubations containing the crude cytosolic fraction, is caused by its consumption with catechol 1,2-dioxygenase. 


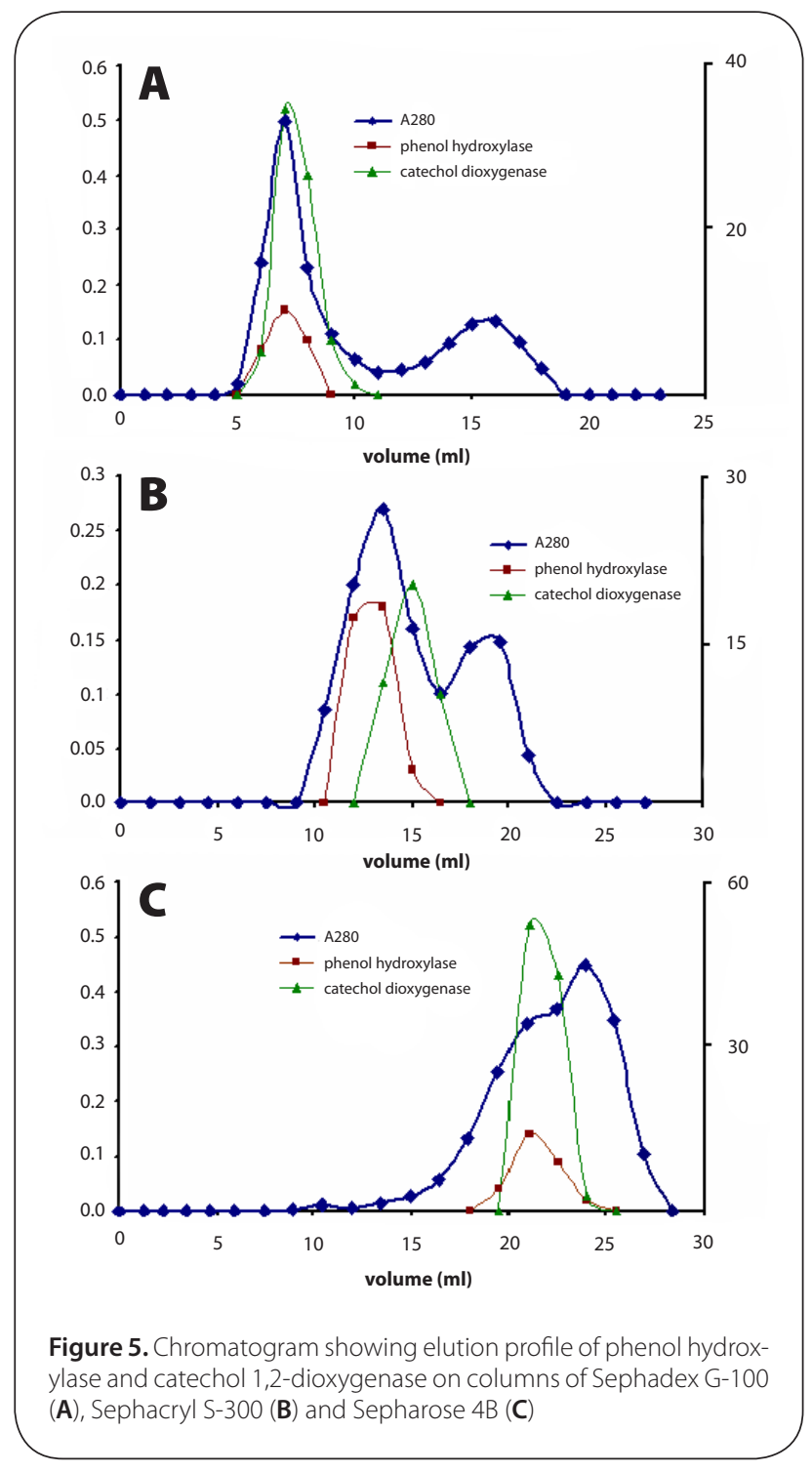

NADPH-dependent phenol hydroxylase was purified from the cytosolic fraction of C. tropicalis by the procedure consisting of chromatography on DEAE-Sepharose, fractionation by polyethylene glycol 6000 and gel filtration on Sepharose 4B and partially characterized. The $K_{m}$ value of NADPH-dependent phenol hydroxylase of C. tropicalis for phenol is one order of magnitude higher than the value found for the enzyme from yeast Trichosporon cutaneum (Neujahr and Gaal, 1973). Although catechol 1,2-dioxygenase has recently been successfully isolated from C. albicans TL3 (Tsai and Li, 2007), and its activity was detected in $C$. tropicalis (Ahuatzi-Chacon et al., 2004), the enzyme has not been isolated from $C$. tropicalis as yet. Here, we tried to develop a procedure capable of separating catechol 1,2-dioxygenase from phenol hydroxylase, partially purified by chromatography on DEAE-Sepharose. The partial separation of both enzymes was reached using gel permeation chromatography on a column of Sephacryl S-300. Nevertheless, even this chromatographic method did not provide catechol 1,2-dioxygenase in sufficient amounts and purity for its further characterization. Therefore, additional procedures for its isolation are now tested in our laboratory.

In conclusion, the results presented in this paper demonstrate the ability of cytosolic NADPH-dependent phenol hydroxylase and catechol 1,2-dioxygenase of C. tropicalis to metabolize phenol, which is a contaminant of a wide variety of wastewaters. Here we assume that organisms rich in such enzymes might be able to degrade phenol and might be utilized in bioremediation technologies. The data shown in the paper are the first report showing isolation of both NADPH-dependent phenol hydroxylase and catechol 12,-dioxygenase from $C$. tropicalis and demonstrate the progress in resolving the enzymes responsible for the first steps of phenol degradation by the C. tropicalis strain.

\section{Acknowledgement}

The work is supported by the Grant Agency of the Czech Republic (grants 203/06/0329) and the Ministry of Education of the Czech Republic (grants MSM0021620808 and 1M0505).

\section{REFERENCES}

Ahuatzi-Chacon D, Ordorica-Morales G, Ruiz-Ordaz N, Cristiani-Urbina E, Juarez-Ramirez C, Galindez-Mayer J. (2004) Kinetic study of phenol hydroxylase and catechol 1,2-dioxygenase biosynthesis by Candida tropicalis cells grown on different phenolic substrates. World J. Microbiol Biotechnol 20 : 695-702.

Bastos AER, Tomisielo VL, Nozawa SR, Trevors JT, Rossi A. (2000) Phenol metabolism by two microorganisms isolated from Amazonian forest soil samples. J. Industr. Microbiol. Biotechnol. 24: 403-409.

Bayly RC, Wigmore GJ. (1973) Metabolism of phenol and cresols by mutants of Pseudomonas putida. J Bacterio/ 113: 1112-1120.

Briganti F, Pessione E, Guinta C, Scozzafava A. (1997) Purification, biochemical properties and substrate specificity of a catechol 1,2-dioxygenase from a phenol degrading Acinetobacter radioresistens. FEBS Lett 416: 61-64.

Chang YH, Li CT, Chang MC, Shieh WK. (1998) Batch phenol degradation by Candida tropicalis and its fusant. Biotechnol Bioeng 60: 391-395.

Eck R, Bettler J (1991) Cloning and characterization of a gene coding for the catechol 1,2-dioxygenase of Acinetobacter sp. mA3. Gene 123: 87-92.

Gurujeyalakshmi G, Oriel P. (1989) Isolation of phenol-degradation Bacillus stearothermophilus and partial characterization of phenol hydroxylase. Appl Environ Microbio. 55: 500-502

Komárková E, Páca J. (2000) Kinetics of phenol oxidation by Candida tropicalis yeast (in Czech). Chem Listy 94: 729.

Komárková E, Páca J, Klapková E, Stiborová M, Soccol CR, Sobotka M. (2003) Physiological changes of Candida tropicalis population degrading phenol in fed batch reactor. Brazil Arch Biol\& Technol 46: 537-542.

Krug M, Ziegler H, Straube G. (1985) Degradation of phenolic compounds by yeast Candida tropicalis HP15. I. Physiology of growth and substrate utilization. J Basic Microbio/ 2: 102-110.

Krug M, Straube G. (1986) Degradation of phenolic compounds by yeast Candida tropicalis HP15. II. Some properties of the first two enzymes of the degradation pathway. J Basic Microbio/ 5: 271-281.

Martius GGS, Stottmeister U, Jechorek M, Páca J. (1996) Inhibition concentration of phenolic substances under different cultivation conditions. Part II. Impact of dissolved oxygen concentration and temperature on degradation kinetics. Acta Hydrochim Hydrobio/ 24: 168-175.

Nakai C, Horiike K, Kuramitsu S, Kagamiyama H, Nozaki M, (1990) Three isoenzymes of catechol 1,2-dioxygenase (pyrocatechase), aa, $a \beta$, and $\beta \beta$, from Pseudomonas arvilla (-1. J Biol Chem 265: 660-665. 
Neujahr HY, Gaal A. (1973) Phenol hydroxylase from yeast. Purification and properties of the enzyme from Trichosporum cutaneum. Eur J Biochem $\mathbf{3 5}$ : 386-400.

Páca J, Komárková E, Prell A, Stiborová M, Sobotka M. (2002) Kinetics of phenol oxidation by Candida tropicalis: effects of oxygen supply rate and nutrients on phenol inhibition. Folia Microbio/ 47: 685-692.

Páca J Jr, Kremláčková V, Turek M, Suchá V, Vilímková L, Páca J, Halecký M, Stiborová M, (2007) Isolation and partial characterization of cytoplasmic NADPHdependent phenol hydroxylase oxidizing phenol to catechol in Candida tropicalis yeast. Enzyme Microb Tech 40: 919-926.

Páca J, Martius GGS. (1996) Inhibition concentration of phenolic substances under different cultivation conditions. Part I. Phenol oxidation by mixed microbial population in a model system. Acta Hydrochim Hydrobio/ 24: 127-131.

Shen XH, Liu ZP, Liu SJ. (2004) Functional identification of the gene locus (ncg12319) and characterization of catechol 1,2-dioxygenase in Corybebacterium glutamicum. Biotechnol Lett 26: 575-580.

Stephenson T. (1990) Substrate inhibition of phenol oxidation by a strain of Candida tropicalis. Biotechn Lett 12: 843-846.

Stiborová M, Asfaw B, Frei E, Schmeiser HH, Wiessler M. (1995) Benzenediazonium ion derived from Sudan I forms an 8-(phenylazo)guanine adduct in DNA. Chem Res Toxico/ 8: 489-498.

Stiborová M, Frei E, Wiessler M, Schmeiser HH. (2001a) Human enzymes involved in metabolic activation of carcinogenic aristolochic acids: evidence for reductive activation by cytochromes P450 1A1 and 1A2. Chem Res Toxicol 14: 1128-1137.
Stiborová M, Hájek M, Vošmiková H, Frei E, Schmeiser HH. (2001b) Isolation of DT-diaphorase [NAD(P)H dehydrogenase (quinone)] from rat liver cytosol: identification of new substrates, carcinogenic aristolochic acids. Collect Czech Chem Commun 66: 959-972.

Stiborová M, Hudeček J, Páca J Jr, Martínek V, Páca J. (2004) Study of enzymes metabolizing environmental pollutants as a means of modulating their biodegradation (in Czech). Chem Listy 98: 876-890.

Stiborová M, Suchá V, Mikšanová M, Páca J Jr, Páca J. (2003) Hydroxylation of phenol to catechol by Candida tropicalis: involvement of cytochrome P450. Gen Physiol Biophys 22: 167-179.

Tsai S-C, Li Y-K. (2007) Purification and characterization of a catochel 1,2-dioxygenase from a phenol degrading Candida albicans TL3. Arch Microbio/ 187: 199-206.

Tsai S-C, Tsai L-D, Li Y-K. (2005) An isolated Candida albicans TL3 capable of degrading phenol at large concentration. Biosci Biotechnol Biochem 69 : 2358-2367.

Yang R, Humphrey AE. (1975) Dynamic and steady state studies of phenol biodegradation in pure and mixed cultures. Biotechno. Bioeng 17: 1211-1235.

Xu D, Ballou DP, Massey V. (2001) Studies of the mechanism of phenol hydroxylase: mutants Tyr289Phe, Asp54Asn, and Arg281Met. Biochemistry 40 12369-12378. 